\title{
Des greniers ruraux aux greniers militaires et urbains. Les enjeux historiques d'une enquête archéologique
}

\author{
Michel Reddé
}

Comme l'a souligné à juste titre Stéphane Martin dans l'introduction de cet ouvrage, le regain d'intérêt actuel pour les structures de stockage de l'Antiquité n'est pas seulement une lubie d'archéologues en mal de classement typologique$^{1}$. Il ne s'agit rien moins, en effet, que de tenter d'aborder des questions aussi complexes que la capacité productive des campagnes à un moment donné du temps, mais aussi celle des circuits de distribution. J'englobe dans ce terme à la fois le marché libre mais aussi les réquisitions, la fiscalité et la question des réseaux, privés, publics, commissionnés qui gèrent les relations entre producteurs et consommateurs. Or ces derniers ne sont pas tous égaux : l'armée, les centres urbains, grands ou petits n'ont pas nécessairement les mêmes besoins de ravitaillement et n'obéissent pas forcément aux mêmes logiques économiques.

Jusqu'à une époque récente, la question du stockage a surtout intéressé les historiens de l'époque romaine, notamment les spécialistes du monde méditerranéen, préoccupés de comprendre comment fonctionnait l'annone de la mégapole romaine, un problème qui a focalisé l'attention et détourné le regard des régions périphériques de l'Empire, particulièrement celles de l'Europe tempérée ${ }^{2}$. Les difficultés croissent en effet à mesure qu'on s'éloigne des secteurs pour lesquels on ne dispose plus de sources textuelles ou épigraphiques et où toute l'interprétation repose sur l'archéologie, car même les enquêtes de terrain les plus sophistiquées ne sont pas toujours capables de nous apporter des informations très précises sur la nature des denrées produites dans les campagnes et conservées dans des entrepôts souvent arasés jusqu'aux fondations. On peut essayer de rappeler brièvement quelques-uns des problèmes qui se posent à nous.

1 Je ne reprends pas ici l'historique de la question ni l'exposé des problématiques, tels qu'ils ont été énoncés dans ce texte introductif.

2 C'est ce que l'on constate toujours quand on consulte la base en ligne de l'ANR Entrepôts (<http://www.entrepots-anr.efa.gr >, consulté le 7 juin 2018). 
a. En Gaule, notre information sur les structures de stockage est nettement meilleure dans les zones de production que dans les zones de consommation urbaine. Seuls font exception les greniers militaires, relativement mieux connus, qui nous permettent d'évaluer les besoins propres de l'armée. Mais que savons-nous, par exemple, des entrepôts d'une capitale provinciale comme Cologne, a fortiori de chefs-lieux de cités comme Saint-Quentin, Metz, Langres ... ? Et ne parlons pas des agglomérations secondaires ${ }^{3}$. À l'inverse, en Italie, et notamment à Rome, à Ostie ou dans le golfe de Naples, notre connaissance des capacités de stockage destinées à la consommation urbaine est bien supérieure, alors que celle des établissements ruraux n'est pas la mieux étudiée.

b. Comment apprécier la capacité productive des campagnes de la Gaule indépendante ? Lorsque, sur un établissement de l'âge du Fer, nous mettons au jour un petit nombre de greniers sur poteaux, avons-nous affaire à la totalité de la récolte ou seulement à la part réservée pour la consommation domestique et les semences de l'année suivante ? La question peut en effet se poser depuis que nous connaissons l'existence, pour l'instant encore très exceptionnelle, de sites concentrant de nombreuses petites unités d'entrepôts, comme c'est le cas à Entrammes, dans la Mayenne, mais dont la totalité des structures est loin d'être négligeable puisqu'on atteint une superficie globale d'environ $720 \mathrm{~m}^{2}$ (Guillier et al. 2015; voir dans le présent volume la fig. 5.11 et les p. 102-104). On peut en effet s'interroger sur le rôle les agglomérations ouvertes de la Tène $\mathrm{C}$ et des oppida de la Tène finale dans la centralisation des denrées alimentaires produites dans les campagnes environnantes. On doit aussi évoquer le rôle des fermes dites "aristocratiques" de l'Ouest de la Gaule, où la capacité de stockage semble significative mais n'est pas nécessairement le signe assuré d'une possession individuelle : une propriété collective n'y est en effet pas exclue. La plupart du temps, nous ignorons donc sur quelle base foncière et sur quel type d'exploitation repose la quantité de produits conservés dans un endroit donné (voir supra Chapitre 4).

c. Une question exactement inverse se pose lorsque, à l'époque romaine, nous rencontrons l'existence de greniers à très grande capacité au sein d'un site rural, comme c'est le cas à Bad Rappenau "Maueräcker" (cat. 251) ou Bad Rappenau "Babstadt", près de Heilbronn (Baden-Württemberg, DE), ou encore à Biberist ( $\mathrm{CH}$; cat. 85). Dans le premier cas, les auteurs, partant de l'hypothèse d'un domaine de 100 ha autour de la villa ${ }^{4}$, considèrent que ce grand horreum

3 Dans son dernier ouvrage, Javier Salido Domínguez en a dressé, pour les Gaules et les Germanies, une liste courte mais le mélange des types de structures, d'agglomérations, de dates permet justement de constater combien la question est mal connue (Salido Domínguez 2017, 124-32).

4 Elle n'est fondée que sur l'absence d'autres vestiges connus autour de la villa. 
de $608 \mathrm{~m}^{2}$, sans doute à deux étages, si on prend en compte la présence de ses contreforts, est trop vaste pour la production d'un seul domaine, et ils concluent à une fonction de stockage central pour plusieurs établissements ruraux (Hartmann et Meyer 2001; Wulfmeier et Hartmann 2009). Cette hypothèse a fait école : dans un article paru récemment, Matthieu Poux et ses collaborateurs proposent ainsi, à partir de l'analyse du vaste granarium de Panossas (Isère), de reconstituer un système d'approvisionnement annonaire à destination du limes de Germanie en s'appuyant sur l'existence d'un réseau d'horrea à grande capacité situés près des grandes voies du réseau d'Agrippa, depuis le nord de la Narbonnaise jusqu'à la mer du nord (Poux 2017 ; cat. 134). Nous aurons à revenir sur cette question fondamentale : sommes-nous, dans ces cas-là, face à des greniers publics installés au centre d'une zone rurale pour collecter localement les denrées destinées au ravitaillement militaire, ou devant des latifundia privés, ou encore des domaines impériaux, pourvus de gigantesques horrea?

d. La dernière question, qui a été longuement posée tout au long de cet ouvrage, est celle du volume de stockage de tel ou tel type de bâtiment, en fonction de son architecture mais aussi de son mode d'entrepôt des grains. Notre évaluation de la capacité productive des campagnes, de la superficie de l'ager cultivé dépend largement de la manière dont nous calculons cette contenance, un exercice dans lequel, il faut bien l'avouer, entrent une bonne part de spéculations et d'a priori historiques. Pour ne pas revenir une nouvelle fois sur ces calculs souvent aléatoires, nous aborderons ici la question en considérant seulement l'emprise au sol des bâtiments, sachant que celle-ci peut être doublée par l'adjonction d'un étage, et sans tenir compte du mode de stockage, en vrac, en caissons, en tas ou en sacs. Nous prendrons essentiellement en compte la différence relative de superficie, à technique identique de dépôt des mêmes produits.

Pour ce faire, nous avons volontairement sélectionné ici différents greniers empruntés respectivement au monde militaire, urbain et rural, afin d'en comparer les écarts, mais en conservant dans un tableau les volumes estimés par les auteurs et qui peuvent considérablement varier selon le mode d'entrepôt supposé (Tabl. 7.1, p. 142-144). On ne prend en compte, pour simplifier, que l'hypothèse d'un stockage des grains, tout en sachant bien que d'autres denrées pouvaient être conservées dans ces bâtiments (les données chiffrées d'estimation volumétriques sont empruntées à Blöck 2011-2012; Demarez et al. 2010 ; Kehne 2008 ; Poux 2017 ; Schubert 2016 ; Schucany 2006 ; Willems 1988).

On constate que la contenance des mêmes greniers diffère très sensiblement d'un auteur à l'autre, selon le mode de stockage et/ou l'architecture que l'on reconstitue, ce qui explique que nous ne prenions ici en compte que la superficie. En ne considérant que ce critère et à mode de stockage identique, 


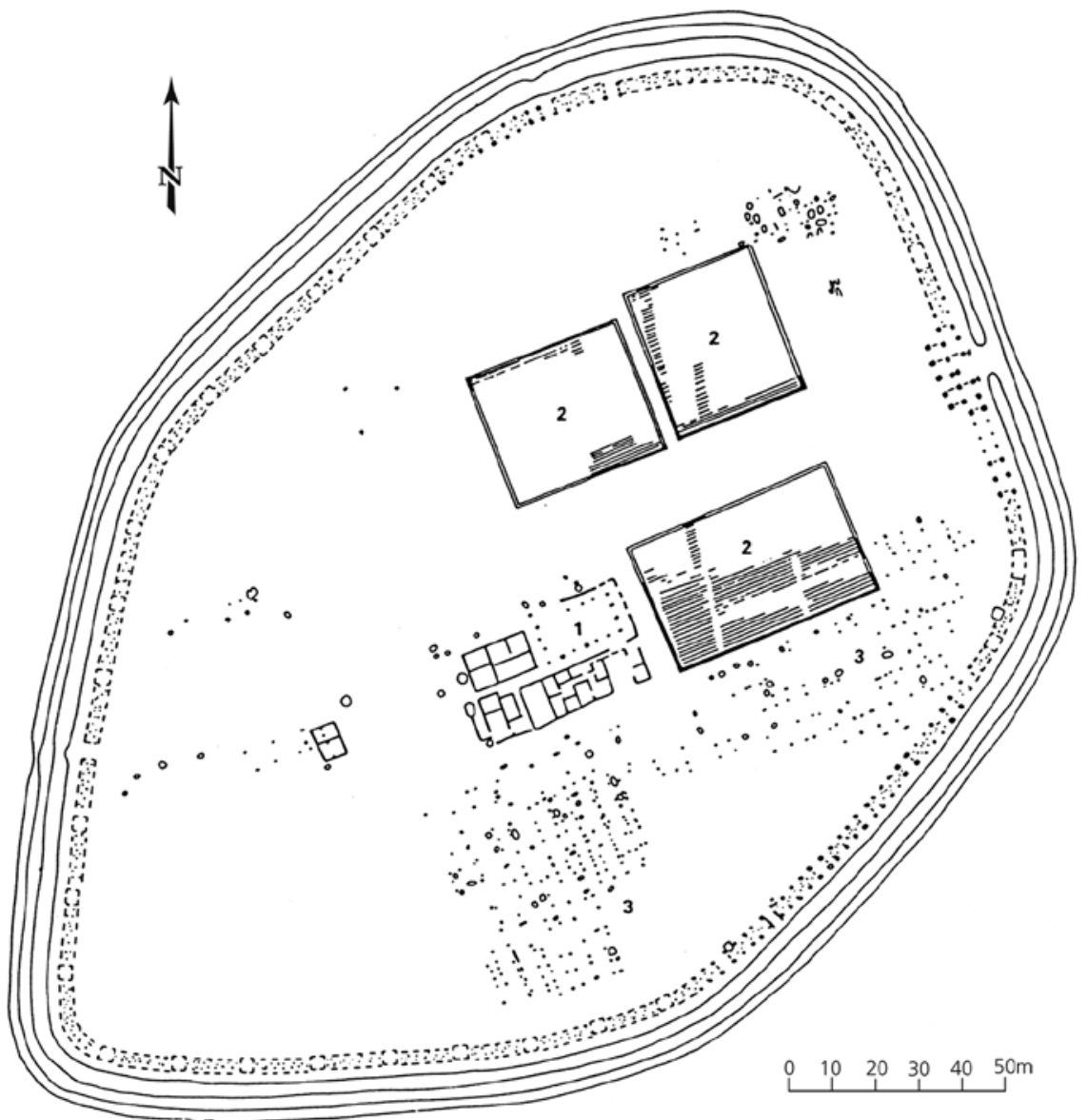

FIGURE 7.1 Le camp de Rödgen (Bad Nauheim, Hesse) et ses grands horrea (2) REDDÉ ET AL. 2006, 217

les écarts entre les grands sites militaires de la conquête de la Germanie et les plus grandes villae connues sautent immédiatement aux yeux.

Les greniers de Rödgen, avec trois horrea occupant une superficie de $3457 \mathrm{~m}^{2}$ (fig. 7.1), sont au moins 5,85 fois plus grands que celui de la villa de Biberist, 9,21 fois plus vastes que celui de Voerendaal (cat. 33). Le camp de Rödgen est généralement considéré comme lié aux opérations de Drusus, en 11-9 av. n. è. L'énorme "Horreastadt" d'Anreppen, sans doute mise en place pour la campagne de 9 de n. è., n'atteint pas cette capacité, avec "seulement" $2370 \mathrm{~m}^{2}$, ce qui reste fort respectable, surtout si l'on y ajoute l'autre grand magasin à cour (fig. 7.2-3). En revanche un camp légionnaire classique comme celui de Neuss "Koenenlager", au II ${ }^{\mathrm{e}}$ s. de n. è., possède des greniers d'une 


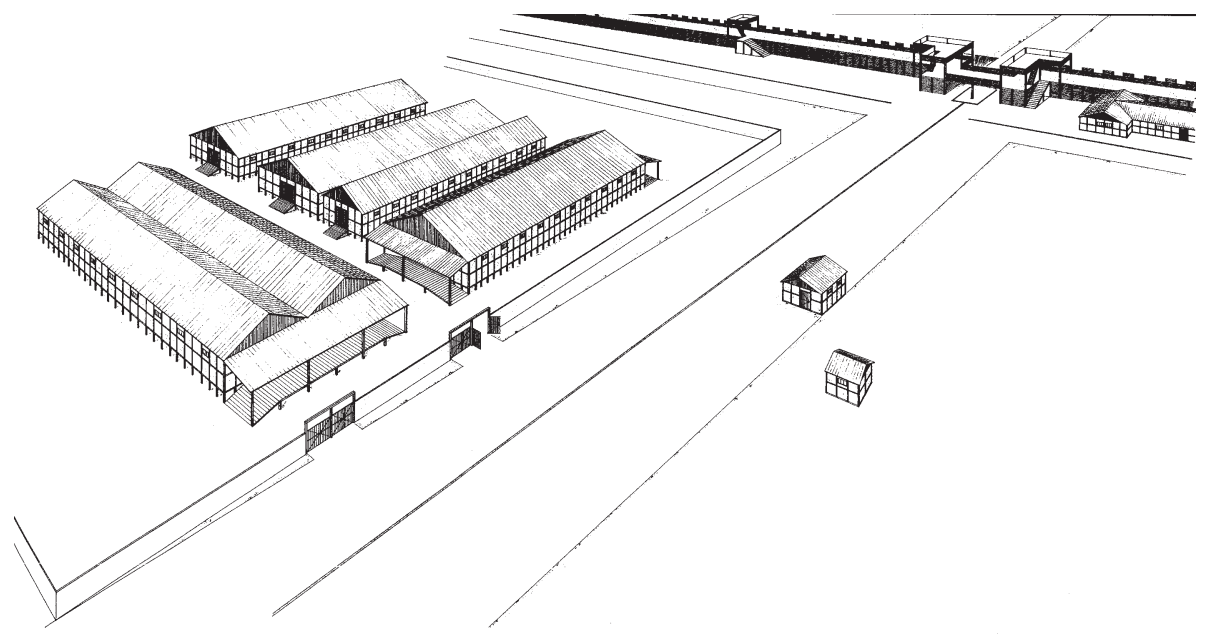

FIGURE 7.2 Delbrück/Anreppen : restitution de l'“Horreastadt" par J.-S. Kühlborn REDDÉ ET AL. 2006, 111

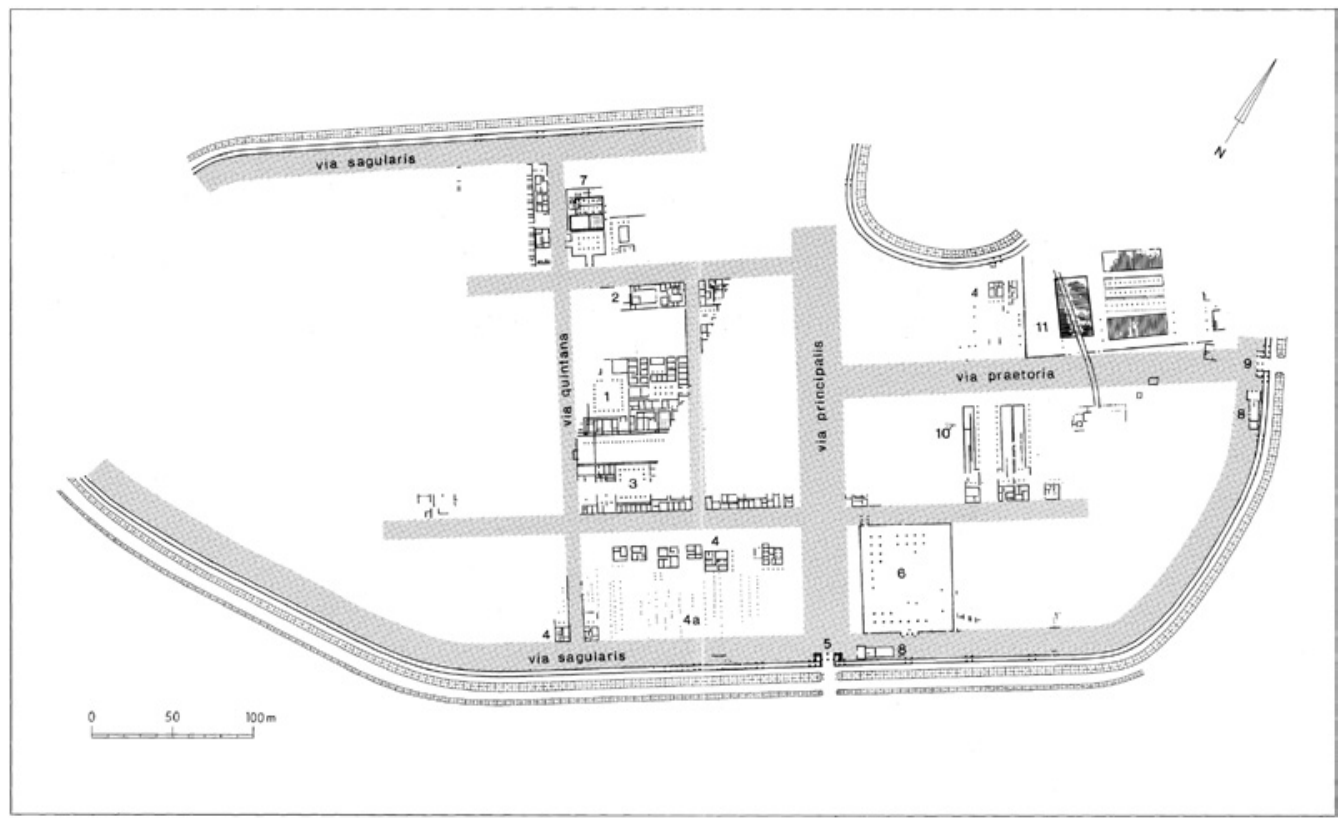

FIGURE 7.3 Delbrück/Anreppen : plan général, par J.-S. Kühlborn REDDÉ ET AL. 2006, 261 
superficie de $858 \mathrm{~m}^{2}$ soit 1,5 fois la capacité de Bad Rappenau "Maueräcker" ou de Biberist.

Les camps auxiliaires disposent naturellement de capacités inférieures, mais parfois très hautes si on les compare à celles des castra légionnaires : ainsi est-on étonné de la taille des horrea de la Saalburg $\left(489 \mathrm{~m}^{2}\right)$ quand on la confronte à celle du "Koenenlager", dont l'effectif théorique était pourtant 10 à 12 fois supérieur. À Walheim II, dans la vallée du Neckar, le camp de numerus initial semble avoir été transformé en base logistique régionale, avec la construction de très vastes greniers, successivement $\mathrm{G}$ et $\mathrm{F} 1$, beaucoup trop grands pour satisfaire aux seuls besoins de la garnison restante (fig. 7.4). Bien clairement, même à une époque où les grandes expéditions de conquête avaient cessé, l'entrepôt des denrées nécessaires au ravitaillement militaire n'obéissait donc pas toujours à des lois de proportionnalité avec la taille de la garnison. On peut en outre supposer que le système avait passablement évolué au cours du temps : ainsi, dans le camp A d'Oedenburg, daté entre Claude et la fin du règne de Néron, l'horreum fouillé est fort limité (environ $48 \mathrm{~m}^{2}$ ) même s'il est accompagné, comme c'est souvent le cas, d'un magasin à cour beaucoup plus vaste, servant aussi au stockage de denrées autres que les grains (Reddé 2009, 120). La superficie probable du camp (sans doute autour de 2,5 ha) le place dans la catégorie supérieure des camps claudiens comparables, sans qu'on puisse affirmer qu'il s'agit d'un cantonnement auxiliaire car sa garnison semble mixte (Reddé 2009, 110-11). Les horrea d'Oberstimm, sur le Danube, sont nettement plus vastes $\left(117 \mathrm{~m}^{2}\right)$, alors que la superficie globale du castellum est plus faible (1,63 ha).

Ces différentes données doivent être confrontées avec celles d'établissements de la Tène finale dont la capacité totale de stockage est connue. À Jaux, en Picardie, par exemple, les trois greniers sur poteaux occupent une superficie d'environ $24 / 25 \mathrm{~m}^{2}$, auxquels il faut ajouter la contenance d'un silo pouvant accueillir environ 2,8 $\mathrm{t}$ de blé, capable de nourrir 11 personnes pendant un an, soit la population probable de la ferme (Malrain et al. 1996). Si cet ensemble représente bien celui de toute la production annuelle, et en prenant pour hypothèse que le silo suffit à la consommation domestique (mais l'hypothèse inverse n'est pas exclue) cela implique qu'il aurait fallu environ 135 à 145 fermes identiques pour remplir les greniers de Rödgen, à hauteur et à mode de stockage identiques. Comme il n'était naturellement pas pensable de ne rien laisser aux producteurs pour se nourrir et ensemencer l'année suivante, on peut mesurer l'impact et l'extension régionale des prélèvements opérés sur les campagnes de Gaule intérieure pour fournir les copiae d'une partie de l'armée de Germanie, au début de la conquête. Dans le cas de Rödgen, il ne s'agit que d'une seule base logistique et nous ne savons pas si elle suffisait au ravitaillement de toute l'expédition. 


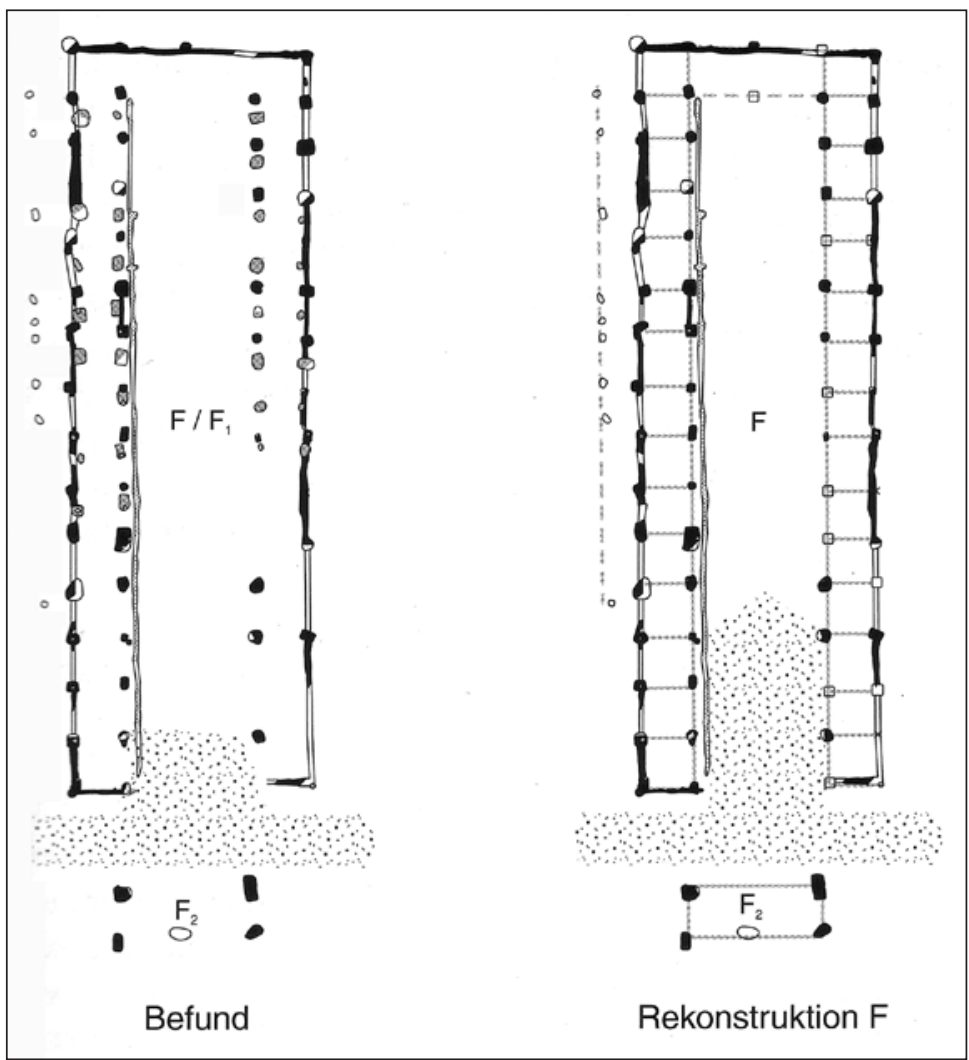

FIGURE 7.4 Le bâtiment F de Walheim, d'après K. Kortüm REDDÉ 2011A, 127

Comparé à la superficie du grenier de la petite villa de Hambach 111 (cat. 97) dans les lœss fertiles des Braunkohlenrewier, soit 37,5 $\mathrm{m}^{2}$, on ne constate pas de saut quantitatif important par rapport à Jaux qui comprend en outre un important silo. Les autres établissements de cette région, de taille généralement moyenne, disposent certes de capacités de stockage supérieures, mais celles-ci sont très inférieures à celles des très grands horrea qu'on a l'habitude de citer, et elles se rapprochent plus certainement de la réalité. En outre, on ne sait pas toujours bien dater ces greniers de manière précise et leur évolution spatiale dure parfois jusqu'au III ${ }^{\mathrm{e}}$ s. de n. è. (Schubert 2016).

Bien sûr ces évaluations et ces comparaisons sont probablement fausses dans l'absolu, d'autant qu'elles ne concernent pas des sites contemporains. Elles ne sont donc proposées ici que pour fournir un ordre de grandeur raisonnable, à partir d'une base de calcul homogène, et permettre de réfléchir à ce 
qu'implique, en termes économiques, la superficie d'une structure de stockage. L'archéologie observe par ailleurs une tendance continue à l'agrandissement des greniers ruraux, tout au long de l'Empire. Diderick Habermehl (2013) en a fourni une illustration suggestive à l'aide d'une double planche que nous reproduisons ici (fig. 7.5). Toutefois, cette planche peut conduire à des interprétations discutables si nous n'y prenons garde. L'accroissement de la superficie des greniers de Seclin, par exemple, signifie-t-elle une augmentation productive de l'ordre de 1 à 60 ? Évidemment non, sauf si l'on admet un accroissement considérable de la surface cultivée, donc une transformation de la structure foncière et probablement un phénomène de concentration. Dans le cas de Alle (cat. 125 et 221), mentionné plus haut dans le tabl. 1, il est peu probable qu'un même terroir ait pu, dans les conditions techniques relativement stables de l'Antiquité, passer d'une production stockable dans un grenier de $48 \mathrm{~m}^{2}$, au début du $\mathrm{I}^{\mathrm{er}} \mathrm{s}$. de n. è., à une capacité près de 15 fois supérieure, deux siècles plus tard. Cette modification considérable traduit évidemment autre chose qu'un accroissement des rendements et de productivité.

Les greniers urbains, en Gaule du nord, restent malheureusement mal connus. Les plus récemment fouillés et publiés, ceux de la rue Maucroix à Reims, ne sont pas dégagés dans leur totalité, de sorte que leur superficie totale est le fruit d'une estimation (fig. 7.6 et 7.7). On doit les confronter à ceux de Trèves, datables du début de l'Antiquité tardive, à un moment où cette ville devenait la capitale de la Gaule ; ils occupent une superficie de $2800 \mathrm{~m}^{2}$ (Merten 2005). Il est donc douteux que ceux de Reims, au $\mathrm{I}^{\mathrm{er}} \mathrm{s}$., aient pu atteindre une superficie au sol de près de $3250 \mathrm{~m}^{2}$, comme le propose Maxence Poirier (2011; ici fig. 7.8). On serait là, en effet, dans une dimension proche de celle des horrea des grandes bases logistiques des campagnes de Germanie, destinées à approvisionner plusieurs dizaines de milliers d'hommes pendant des mois et supérieure à celle des horrea de Trèves.

Le cas des grands entrepôts mis au jour par Éric Binet à Amiens, toujours inédits, n'est pas moins douteux : les fouilleurs restituent une série de 10, voire 11 édifices, construits vers 80 de $n$. è., précédés par des portiques, et constituant des îlots (fig. 7.9). Mais il s'agit là d'une proposition hasardeuse, compte tenu de l'état de conservation réel des vestiges dont une grande partie est totalement arasée : la superficie restituée - $3000 \mathrm{~m}^{2}$ - en ferait les plus grands de notre série et les situerait au niveau des grands entrepôts romains, une hypothèse sans doute excessive pour la petite ville qu'était Amiens au I ${ }^{\mathrm{er}} \mathrm{s}$. : ainsi, à Rome, les horrea Agrippiana offrent-ils une dimension de $275^{0} \mathrm{~m}^{2}$, les horrea Lolliana de $2500 \mathrm{~m}^{2}$ (Bernardos Sanz et Virlouvet 2016, 82). L'identification comme greniers n'est d'ailleurs pas absolument certaine, bien que possible, mais ce n'est pas le lieu de discuter ici des autres hypothèses possibles. À l'autre 
Storage building development trajectories

Time $\longrightarrow$

Hambach $5^{12}$

罳

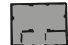

Hambach 127

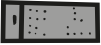

Jülich-Kirchberg (WW 112)

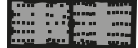

Voerendaal-Ten Hove

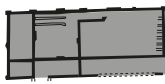

Oss-Westerveld

$\vdots$

Houten-Tiellandt

㺊

Tiel-Passewaaij

:.

:

Rijswijk-De Bult

i.1․

FIGURE 7.5 Évolution des horrea ruraux en Gaule du nord D'APRÈS HABERMEHL 2013, 149-50 FIG. 5 


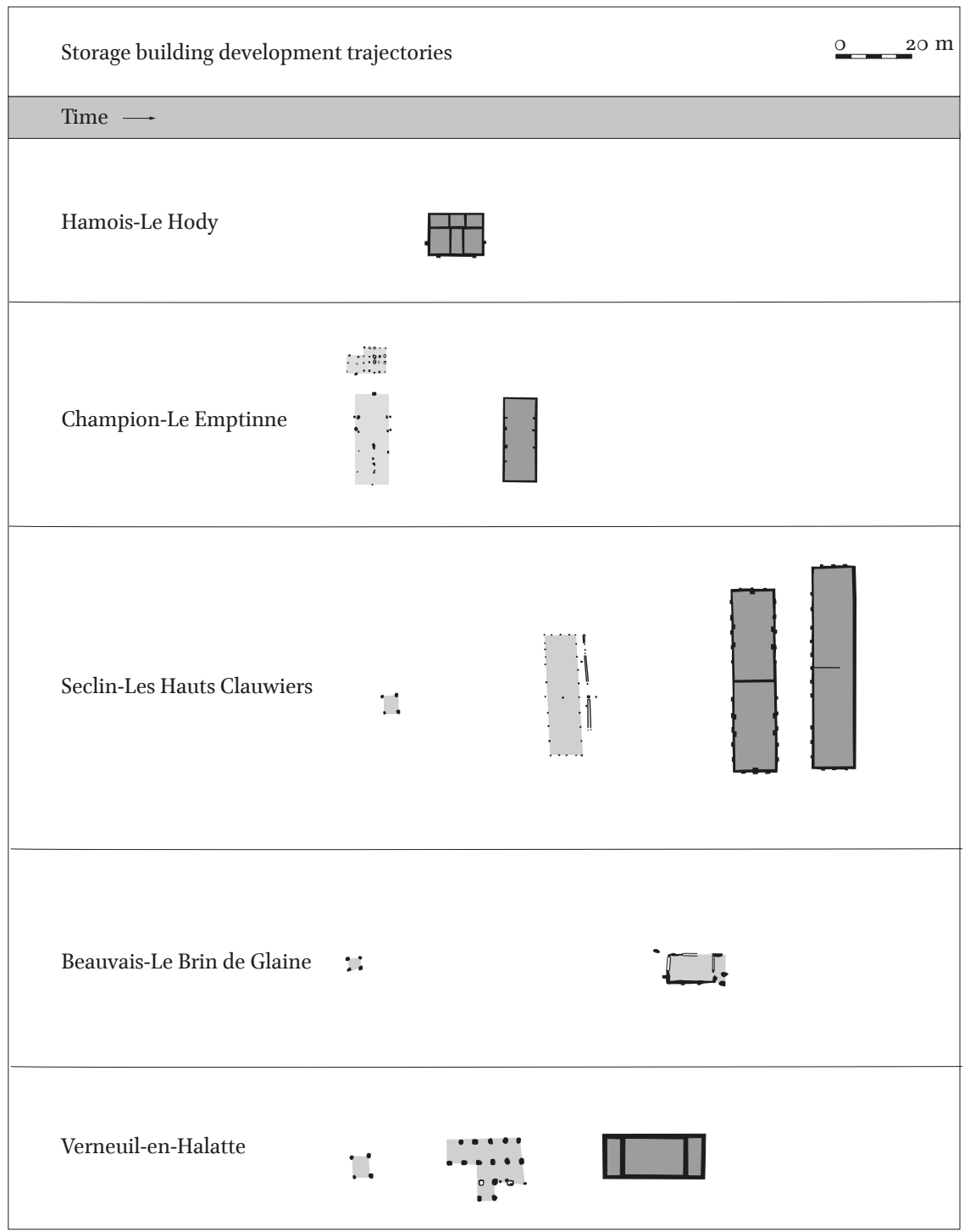

FIGURE 7.5 (cont.) 
A $80,00 m_{-} \Psi_{-\ldots}$ B 80,00 m

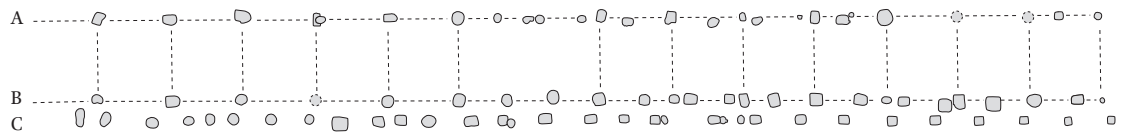

Us.1168

$\mathrm{C} 80,00 \mathrm{~m}$
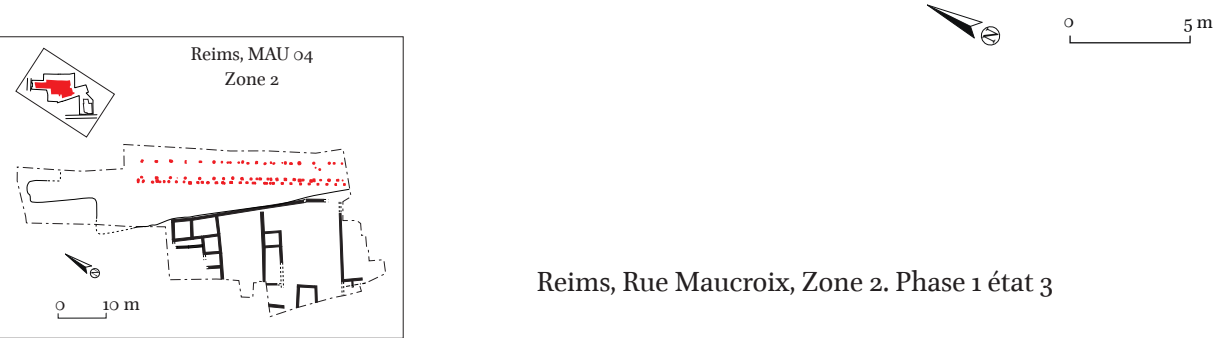

FIGURE 7.6 Les horrea de la rue Maucroix à Reims (état 1)

D'APRÈS ROLLET ET AL. 2011

Reims, Rue Maucroix, Zone 2. Phase 1 état 3
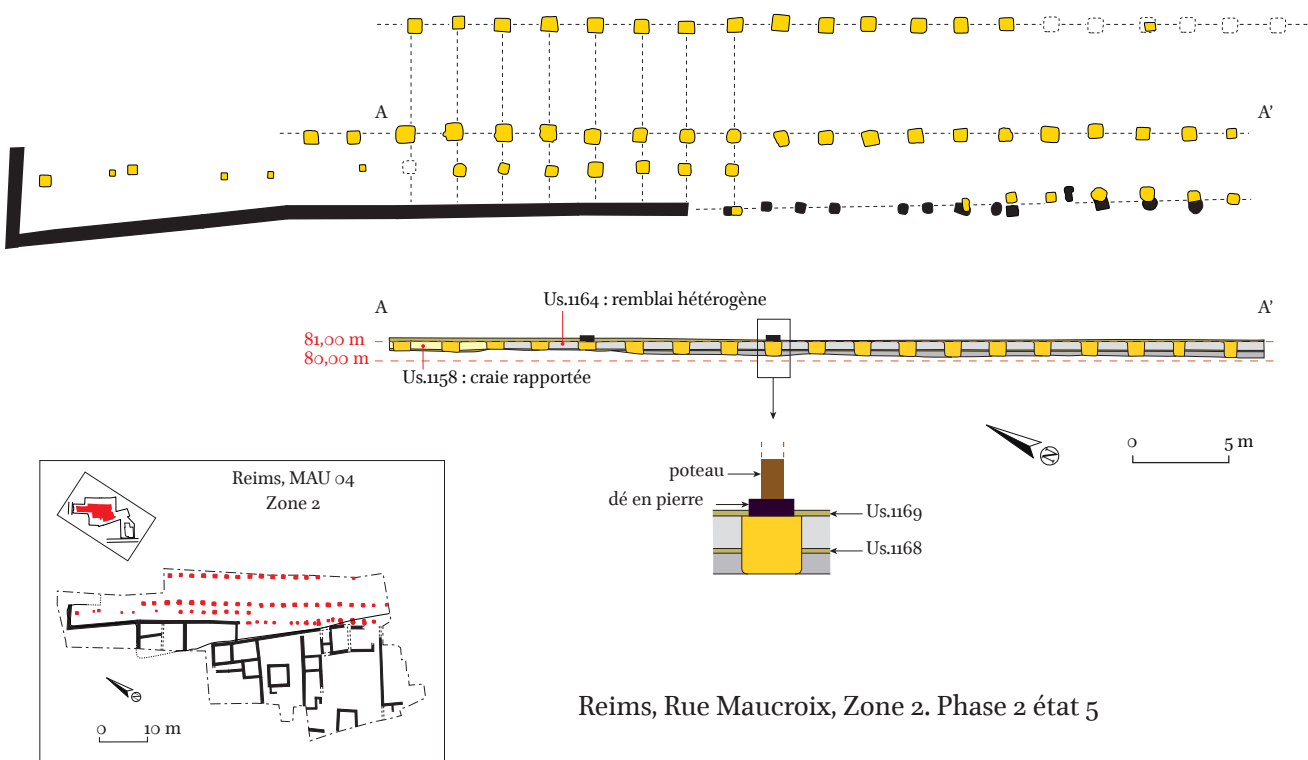

Reims, Rue Maucroix, Zone 2. Phase 2 état 5

FIGURE 7.7 Les horrea de la rue Maucroix à Reims (état 2)

D'APRÈS ROLLET ET AL. 2011 


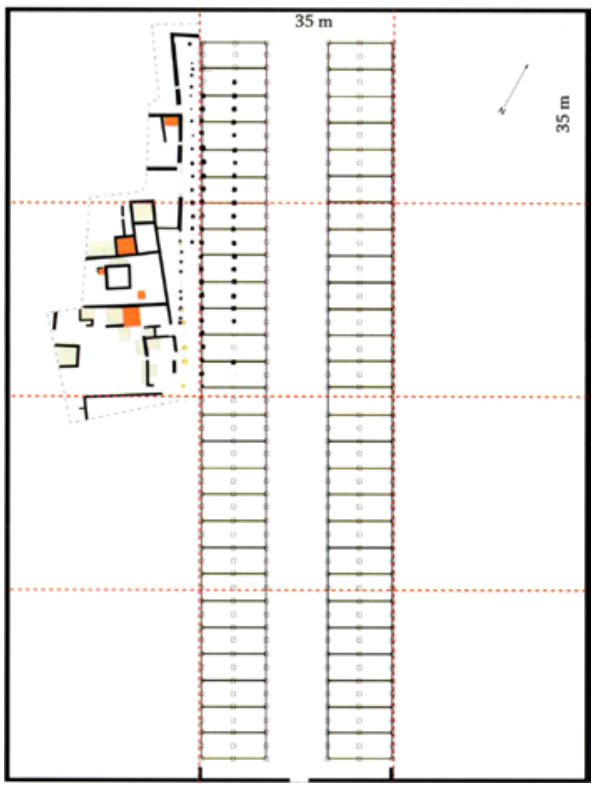

FIGURE 7.8

Les horrea de la rue Maucroix à Reims (état restitué)

D'APRÈS POIRIER 2011, 132

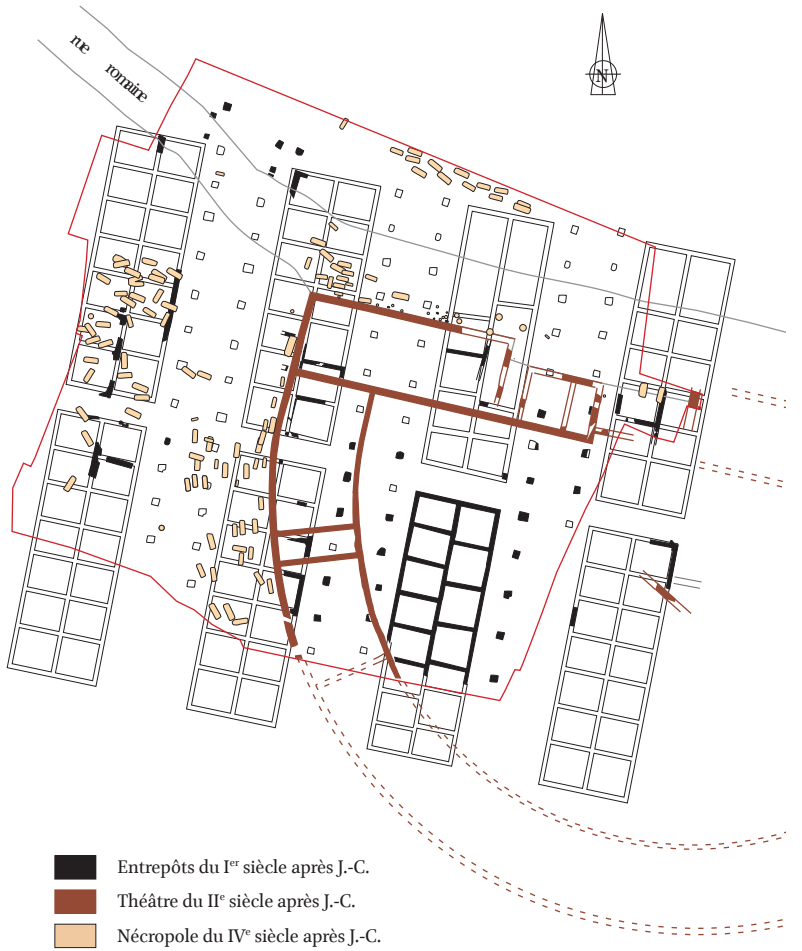

FIGURE 7.9

Les horrea d'Amiens, îlot de la Boucherie D'APRÈS BINET ET PORTZAMPAC 2007, AVEC MODIFICATIONS 
bout de la ville, le petit édifice mis au jour dans la "ZAC Cathédrale" a de bonnes chances, vu sa taille $\left(90 \mathrm{~m}^{2}\right)$ d'être un édifice privé plutôt que public (Matterne, Yvinec, et Gemehl 1998).

Venons-en maintenant, avant de conclure, au "granarium" fouillé par M. Poux et son équipe à Panossas dans l'Isère (Poux 2017). La surface de stockage au sol, une fois soustraite celle du hall central, est d'environ $35 \mathrm{~m}^{2}$, une superficie respectable, certes, mais qui n'est pas très différente de celle de Heitersheim (cat. 254), de Voerendaal ou de Alle (état 2), et parait sensiblement moindre que celle de Biberist, de Alle (état 3), ou de Bad Rappenau. La liste pourrait être allongée. M. Poux propose une datation entre, au plus tôt, le début de l'époque flavienne et le milieu du $\mathrm{III}^{\mathrm{e}} \mathrm{s}$.

À partir de cet exemple récemment fouillé, les auteurs s'appuient sur une carte des découvertes connues de grands horrea pour tenter de montrer l'existence d'un réseau de collecte annonaire, lié aux grands axes du réseau routier de Gaule, à destination des villes et du limes, et ils en publient une carte. Si l'intention est louable et l'hypothèse recevable a priori, la démonstration pêche par l'absence de données chronologiques car le corpus mêle des données de toute nature et de toute époque. On voit ainsi figurer sur la même carte Haltern et Niederbieber, que séparent près de deux siècles. Peut-on, dès lors parler de réseau ? Sans doute de tels systèmes ont-ils bien existé, comme l'a rappelé Marie-Brigitte Carre (Carre 2011; Carre et Laudani 2016). Mais, dans le cas qui nous occupe ici, seule une enquête rigoureuse, avec des séries chronologiquement homogènes et bien datées, permettrait, peut-être, d'asseoir l'hypothèse proposée. Pour l'heure, il paraît sage de ne pas tenter de reconstituer physiquement un tel réseau d'approvisionnement.

Quelques observations s'imposent toutefois au terme de cette enquête dont la prétention n'était certes pas de conclure un débat, mais au contraire de ne pas le clore trop tôt et d'en rappeler les difficultés, d'en mieux cerner les obstacles méthodologiques.

On doit d'abord souligner l'énormité de la demande militaire à l'époque augustéenne, au moment des tentatives successives de conquête de la Germanie. Cet effort exigé des campagnes gauloises a certainement été d'autant plus important que le monde rural protohistorique, riche malgré l'impact inévitable de la guerre des Gaules encore récente, ne pouvait probablement pas fournir instantanément beaucoup plus qu'il ne produisait déjà : à population quasi stable, à conditions techniques identiques, et alors que nous ne voyons pas apparaître, à cette époque, une économie domaniale de type italien capable de fournir un surplus significatif, la demande ne pouvait à elle seule susciter l'offre de manière spontanée (Reddé 2018b). Cette idée que les nouveaux marchés militaires de Germanie suffisaient à provoquer le décollage économique de la Gaule me parait, personnellement, parfaitement anachronique. Il est donc 
clair qu'on doit ici se souvenir de la fameuse phrase de Tacite (Agr. 19.4-5) appliquée, il est vrai, à la Bretagne flavienne : "Par une équitable répartition des charges, il rendait moins dures les prestations de blé et la levée des impôts, en retranchant ces inventions de l'avidité qu'on avait plus de peine à supporter que l'impôt lui-même. Car, par dérision, les Bretons étaient forcés d'attendre près des greniers fermés, et, qui plus est, d'acheter du blé à un prix majoré. Des routes détournées, des régions lointaines étaient assignées aux cités très voisines des quartiers d'hiver, pour porter leurs blés dans d'autres cantonnements écartés et peu accessibles, jusqu'à ce que l'arrangement le plus simple pour tous devînt pour un petit nombre une source de profit"5. Déjà, sous Tibère, les excès des réquisitions avaient conduit à la révolte des Gaules (Reddé 2011b). On voit bien, en comparant la capacité d'un grenier militaire de cette époque avec celle d'une ferme protohistorique, l'énorme différence de capacité qui existe entre ces deux extrémités de la chaîne de ravitaillement.

Que les campagnes gauloises aient progressivement augmenté leur potentiel avec le temps est par ailleurs une évidence. On le voit bien à travers l'évolution de la capacité de stockage des établissements agricoles, mais celle-ci n'est guère visible dans le nord avant, au plus tôt, le dernier tiers du I ${ }^{\text {er }} \mathrm{s}$. de n. è. Les quelques exemples qui montrent des phases successives, comme c'est le cas à Alle, attestent formellement cette progression. On ne saurait pourtant tirer de celle-ci, sans contre-épreuve, l'idée simpliste d'une augmentation équivalente de la production sur un domaine de superficie identique. Derrière l'apparition de ces horrea à grande capacité peuvent en effet se cacher des phénomènes de concentration foncière qui nous échappent totalement. Quant à l'idée de regroupements de stocks destinés au ravitaillement militaire, elle est parfaitement pertinente, notamment quand il s'agit de l'hinterland immédiat du limes, comme c'est le cas de Bad Rappenau, tout près du Neckar. Encore faudrait-il démontrer cette hypothèse de manière précise à travers une enquête plus globale car nous en sommes, pour l'instant, réduits aux spéculations.

Pour finir, les capacités de stockage devraient, dans l'absolu, être évaluées à l'échelle d'un terroir beaucoup plus qu'à celle d'un établissement considéré individuellement, comme le montre l'exemple, pour l'heure exceptionnel, du site protohistorique d'Entrammes, capable de fournir une superficie deux fois plus grande que celle du très grand granarium de Panossas et d'alimenter un camp légionnaire comme le "Koenenlager" de Neuss.... Idée utopique, évidemment, puisque l'archéologie n'a pas les moyens d'évaluer un terroir dans sa totalité.

5 Traduction Les Belles Lettres: Frumenti et tributorum exactionem aequalitate munerum mollire, circumcisis quae in quaestum reperta ipso tributo grauius tolerabantur ; namque per ludibrium adsidere clausis horreis et emere ultro frumenta ac luere pretio cogebantur. Diuortia itinerum et longinquitas regionum indicebantur, ut ciuitates proximis hibernis in remota et auia deferrent, donec quod omnibus in promptu erat paucis lucrosum fieret. 
TABLEAU 7.1 Capacités de stockage de grain estimées pour différents greniers urbains, militaires et ruraux.

\begin{tabular}{|c|c|c|c|c|c|}
\hline & Lieu & $\begin{array}{l}\text { Superficie } \\
\text { au sol }\end{array}$ & $\begin{array}{l}\text { Capacité } \\
\text { estimée }\end{array}$ & $\begin{array}{l}\text { Auteur de } \\
\text { l'estimation }\end{array}$ & Commentaires \\
\hline \multirow{8}{*}{$\begin{array}{l}\text { Sites } \\
\text { militaires }\end{array}$} & $\begin{array}{l}\text { Anreppen, Grand } \\
\text { horreum } \\
\text { à cour }\end{array}$ & $3808 \mathrm{~m}^{2}$ & & \multirow{4}{*}{ Kehne 2008} & $\begin{array}{l}\text { Pas de blé mais autres } \\
\text { produits }\end{array}$ \\
\hline & $\begin{array}{l}\text { Anreppen, } \\
\text { "Horreastadt" }\end{array}$ & $2369,63 \mathrm{~m}^{2}$ & $1640 / 1968 \mathrm{~m}^{3}$ & & $\begin{array}{l}\text { Sacs stockés sur 1,5 m } \\
\text { de haut ( } 1 \text { étage) }\end{array}$ \\
\hline & Anreppen Total & $6177,63 \mathrm{~m}^{2}$ & & & $\begin{array}{l}54 \text { jours de rations de } \\
\text { blé pour expédition de } \\
25 \text { ooo h en } 4 \text { apr. } \\
\text { ( } 1 \text { étage) }\end{array}$ \\
\hline & Rödgen & $3457 \mathrm{~m}^{2}$ & $\begin{array}{l}4149 \mathrm{~m}^{3} \text { dont } \\
3445 \\
\text { pour céréales }\end{array}$ & & $\begin{array}{l}\text { Sacs stockés sur } 1,5 \mathrm{~m} \\
\text { de haut }=7079 \text { rations } \\
\text { annuelles, environ } \\
6 \text { mois de réserve pour } \\
\text { trois légions (cam- } \\
\text { pagnes de Drusus, } \\
11-9 \text { av. n. è.) (1 étage) }\end{array}$ \\
\hline & $\begin{array}{l}\text { Neuss } \\
\text { "Koenenlager" }\end{array}$ & $858 \mathrm{~m}^{2}$ & & \multirow[t]{2}{*}{$\begin{array}{l}\text { Dimensions } \\
\text { calculées } \\
\text { d'après Petriko- } \\
\text { vits } 1975\end{array}$} & \\
\hline & Saalburg & $489 \mathrm{~m}^{2}$ & & & \\
\hline & \multirow[b]{2}{*}{ Walheim II } & Bât. G : $45^{2} \mathrm{~m}^{2}$ & & & Milieu $2^{\mathrm{e}}$ siècle. Les \\
\hline & & $\begin{array}{l}\text { Bât. F1: } 378 / 400 \\
\mathrm{~m}^{2}\end{array}$ & & Reddé 2011a & $\begin{array}{l}\text { bâtiments G et H se } \\
\text { succèdent. }\end{array}$ \\
\hline \multirow{4}{*}{ Sites ruraux } & \multirow[t]{2}{*}{ Voerendaal } & \multirow[t]{2}{*}{$375 \mathrm{~m}^{2}$} & $\begin{array}{l}208,2 / 268,72 \\
m^{3}\end{array}$ & $\begin{array}{l}\text { Blöck } \\
2011-2012\end{array}$ & $\begin{array}{l}\text { Stockage en vrac, dans } \\
\text { caissons, sur 0,55/o,7o } \\
\text { m de hauteur } \\
\text { ( } 1 \text { étage). Évaluation } \\
\text { d'après greniers } \\
17^{\mathrm{e}} / 18^{\mathrm{e}} \mathrm{s} .\end{array}$ \\
\hline & & & $300 / 400 \mathrm{~m}^{3}$ & $\begin{array}{l}\text { Willems 1988, } \\
8-13\end{array}$ & Stockage en vrac \\
\hline & \multirow[t]{2}{*}{ Biberist } & \multirow{2}{*}{400 à $590 \mathrm{~m}^{2}$} & $\begin{array}{l}327,57 / 422,78 \\
\mathrm{~m}^{3}\end{array}$ & $\begin{array}{l}\text { Blöck } \\
2011-2012\end{array}$ & $\begin{array}{l}\text { Stockage en vrac, dans } \\
\text { caissons, sur } 0,55 / 0,70 \\
\text { m de hauteur } \\
\text { ( } 1 \text { étage) }\end{array}$ \\
\hline & & & 1600 à $2000 \mathrm{~m}^{3}$ & Schucany 2006 & $\begin{array}{l}\text { Stockage en caissons, } \\
\text { sur } 4 \text { m de hauteur } \\
\text { ( } 2 \text { étages) }\end{array}$ \\
\hline
\end{tabular}


TABLEAU 7.1 Capacités de stockage de grain estimées pour différents greniers urbains, militaires (cont.)

\begin{tabular}{|c|c|c|c|c|c|}
\hline & Lieu & $\begin{array}{l}\text { Superficie } \\
\text { au sol }\end{array}$ & $\begin{array}{l}\text { Capacité } \\
\text { estimée }\end{array}$ & $\begin{array}{l}\text { Auteur de } \\
\text { l'estimation }\end{array}$ & Commentaires \\
\hline \multirow{13}{*}{ Sites ruraux } & Heitersheim C & $180 \mathrm{~m}^{2}$ & $\begin{array}{l}82,08 / 105,94 \\
\mathrm{~m}^{3}\end{array}$ & \multirow{2}{*}{$\begin{array}{l}\text { Blöck } \\
2011-2012\end{array}$} & Construit avant 100 \\
\hline & Heitersheim $\mathrm{C}_{2}$ & $360 \mathrm{~m}^{2}$ & $\begin{array}{l}158,79 / 204,94 \\
\mathrm{~m}^{3}\end{array}$ & & Vers 120 \\
\hline & Panossas * & $350 \mathrm{~m}^{2}$ & $120 \mathrm{~m}^{3}$ & Poux 2017 & $\begin{array}{l}\text { Stockage en vrac sur } \\
\text { o,3o m ( } 1 \text { étage). } \\
\text { Evaluation minimale }\end{array}$ \\
\hline & Alle phase 1 & $48 \mathrm{~m}^{2}$ & & & Avant 5o/75 de n. è. \\
\hline & Alle phase 2 & $392 \mathrm{~m}^{2}$ & & & Fin $1^{\mathrm{er}} \mathrm{s}$ / / milieu $2^{\mathrm{e}} \mathrm{s}$. \\
\hline & Alle phase 3 & $\begin{array}{l}710 \mathrm{~m}^{2} / \text { surface } \\
\text { de stockage } \\
\text { réelle de } 240 \mathrm{~m}^{2}\end{array}$ & $240 \mathrm{~m}^{3}$ & $\begin{array}{l}\text { Demarez et al. } \\
2010\end{array}$ & $\begin{array}{l}\text { Avant } 25^{0} / 275 \text { de n. è. } \\
\text { Calcul de la surface de } \\
\text { stockage réelle tenant } \\
\text { compte des circulations } \\
\text { internes entre les } \\
\text { caissons avec } \\
\text { tas de } 1 \mathrm{~m} \mathrm{au} \\
\text { maximum }\end{array}$ \\
\hline & $\begin{array}{l}\text { Bad Rappenau } \\
\text { "Maueräcker" } 2^{\mathrm{e}} \\
\text { période }\end{array}$ & $608 \mathrm{~m}^{2}$ & & $\begin{array}{l}\text { Wulmeier et } \\
\text { Hartmann } \\
2009\end{array}$ & \\
\hline & Hambach 111 & $37,5 \mathrm{~m}^{2}$ & $\begin{array}{l}4500-6920 \\
\text { modii }\end{array}$ & \multirow{6}{*}{ Schubert 2016} & $\begin{array}{l}\text { Stockage en sacs } \\
\text { supposé } * *\end{array}$ \\
\hline & Hambach 127 & $112,5 \mathrm{~m}^{2}$ & $\begin{array}{l}13840-20760 \\
\text { modii }\end{array}$ & & $\begin{array}{l}\text { Stockage en sacs } \\
\text { supposé }\end{array}$ \\
\hline & Hambach 130 & $85 \mathrm{~m}^{2}$ & $\begin{array}{l}10188-15688 \\
\text { modii }\end{array}$ & & $\begin{array}{l}\text { Stockage en sacs } \\
\text { supposé }\end{array}$ \\
\hline & Hambach 206 & $5^{8} \mathrm{~m}^{2}$ & $\begin{array}{l}7020-10800 \\
\text { modii }\end{array}$ & & $\begin{array}{l}\text { Stockage en sacs } \\
\text { supposé }\end{array}$ \\
\hline & Hambach 133 & $175 \mathrm{~m}^{2}$ & $\begin{array}{l}21000-32000 \\
\text { modii }\end{array}$ & & $\begin{array}{l}\text { Stockage en sacs } \\
\text { supposé }\end{array}$ \\
\hline & Hambach 303 & $138 \mathrm{~m}^{2}$ & $\begin{array}{l}1663^{2-25584} \\
\text { modii }\end{array}$ & & $\begin{array}{l}\text { Stockage en sacs } \\
\text { supposé }\end{array}$ \\
\hline
\end{tabular}


TABLEAU 7.1 Capacités de stockage de grain estimées pour différents greniers urbains, militaires (cont.)

\begin{tabular}{|c|c|c|c|c|c|}
\hline & Lieu & $\begin{array}{l}\text { Superficie } \\
\text { au sol }\end{array}$ & $\begin{array}{l}\text { Capacité } \\
\text { estimée }\end{array}$ & $\begin{array}{l}\text { Auteur de } \\
\text { l'estimation }\end{array}$ & Commentaires \\
\hline \multirow[t]{4}{*}{$\begin{array}{l}\text { Sites } \\
\text { urbains }\end{array}$} & $\begin{array}{l}\text { Reims, rue } \\
\text { Maucroix }\end{array}$ & $\begin{array}{l}\text { Au moins } 50 \mathrm{~m} \\
\times 5,80 \mathrm{~m}=290 \\
\mathrm{~m}^{2}\left(2^{\mathrm{e}} \text { état }\right)\end{array}$ & & Reddé 2011a & $\begin{array}{l}\text { Début } 1^{\mathrm{er}} \text { s. de n. è. } \\
\text { (phase } 1) \text {; entre } 65-90 \\
\text { et } 90-105 \text { (état } 2 \text { ). } \\
\text { Fouille incomplète. } \\
\text { Restitution de la } \\
\text { superficie dépend de la } \\
\text { restitution. Celle qui est } \\
\text { proposée pour l'état } 2 \\
\text { implique une superficie } \\
\text { totale de } 2 \text { halles de } \\
140 \mathrm{~m} \times 11,6 \mathrm{~m} \text { soit } \\
\text { une emprise au sol de } \\
3248 \mathrm{~m}^{2} \text {. }\end{array}$ \\
\hline & $\begin{array}{l}\text { Amiens, zAC } \\
\text { Cathédrale }\end{array}$ & $\begin{array}{l}20 \mathrm{~m} \times 4,5 \mathrm{~m}= \\
90 \mathrm{~m}^{2}\end{array}$ & & $\begin{array}{l}\text { Matterne, } \\
\text { Yvinec et } \\
\text { Gemehl } 1998\end{array}$ & $2^{\mathrm{e}} \mathrm{s}$. Grenier privé ? \\
\hline & $\begin{array}{l}\text { Amiens, îlot } \\
\text { Boucherie }\end{array}$ & $\begin{array}{l}\text { îlots de } 35 \mathrm{~m} \times \\
10 \mathrm{~m}\end{array}$ & & $\begin{array}{l}\text { Binet et } \\
\text { Portzampac } \\
2007\end{array}$ & Fin $1^{\mathrm{er}} \mathrm{s}$. de n. è. \\
\hline & Trèves & $\begin{array}{l}2 \text { halles de } 70 \times \\
20 \mathrm{~m}= \\
2800 \mathrm{~m}^{2}\end{array}$ & & Merten 2005 & Vers 300 \\
\hline
\end{tabular}

* Panossas est ici classé parmi les sites ruraux, bien que M. Poux semble désormais considérer qu'il puisse s'agir d'un autre type de site.

** L'auteur compte un modius de 8,7 litres ou 8,7 dm³. 4500 modii font donc $39150 \mathrm{l}$ ou $39,15 \mathrm{~m}^{3}$ 